\title{
QUASI-Three-Level Laser Emissions of Neodymium-Doped Disordered Crystal Waveguides
}

\author{
Yang Tan, Feng Chen, Javier Rodríguez Vázquez de Aldana, Haohai Yu, and Huaijin Zhang
}

\begin{abstract}
This paper reports on the quasi-three-level continuous wave laser operation based on waveguide structures in neodymium-doped calcium niobium gallium garnet disordered crystal. Laser wavelength selection through the waveguide cross section was observed. Waveguide structures with different cross sections were fabricated by the ultrafast laser inscription, which have propagation losses around $1 \mathrm{~dB} / \mathrm{cm}$. With suitable pumping conditions, laser emissions were observed at the low wavelengths of $\sim 930$ and $\sim 890 \mathrm{~nm}$. The lasing threshold for the low-wavelength emission was around $50 \mathrm{~mW}$, which is far below the threshold of several watts reported in the bulk laser system. In addition, it was found that the laser generation at the wavelength of $\sim 890 \mathrm{~nm}$ has direct relationship with the volume of the waveguide structure. The results suggest advantages of the waveguide platforms over the bulk systems on the low-wavelength laser emission.
\end{abstract}

Index Terms-Waveguide lasers, disorder laser crystals, femtosecond laser inscription, quasi-three-level lasers.

\section{INTRODUCTION}

A MONG the family of neodymium-doped disordered laser materials, neodymium ion doped calcium niobium gallium garnet $\left(\mathrm{Nd}: \mathrm{Ca}_{3}(\mathrm{NbGa})_{2-\mathrm{x}} \mathrm{Ga}_{3} \mathrm{O}_{12}\right.$ or $\left.\mathrm{Nd}: \mathrm{CNGG}\right)$ is an attractive gain medium. Owing to the random distribution of the niobium and gallium ions, it has lower melting point of $1460{ }^{\circ} \mathrm{C}$, broader absorption band and larger emission bandwidth compared with ordered crystals, which makes it suitable for multi-wavelength and ultra-short laser [1]-[3]. Based on the $\mathrm{Nd}$ :CNGG bulks, laser emissions at the wavelength of $\sim 930 \mathrm{~nm}$ [4], $1.06 \mu \mathrm{m}$ [5] and $1.33 \mu \mathrm{m}$ [6] have been realized.

For the Nd-doped medium, the laser emission with wavelength less than $950 \mathrm{~nm}$ (so-called low-wavelength) could

Manuscript received October 27, 2013; revised May 27, 2014 and July 6, 2014; accepted August 1, 2014. Date of publication August 15, 2014; date of current version September 10, 2014. This work was supported by the National Natural Science Foundation of China under Grant 11274203, the Spanish Ministerio de Ciencia e Innovación under Projects CSD2007-00013 and FIS200909522, and Junta de Castilla y León under Project SA086A12-2. The work of F. Chen and H. Yu was supported by The Fundamental Research Funds of Shandong University under Grant 2014JC002.

Y. Tan and F. Chen are with the School of Physics, State Key Laboratory of Crystal Materials and Key Laboratory of Particle Physics and Particle Irradiation, Ministry of Education, Shandong University, Jinan 250100, China (e-mail: tanyang@sdu.edu.cn; drfchen@sdu.edu.cn).

J. R. Vázquez de Aldana is with the Departamento Física Aplicada, Facultad Ciencias, Universidad de Salamanca, Salamanca 37008, Spain (e-mail: jrval@usal.es).

H. Yu and H. Zhang are with the State Key Laboratory of Crystal Materials, Shandong University, Jinan 250100, China (e-mail: haohaiyu@sdu.edu.cn; huaijinzhang@sdu.edu.cn).

Color versions of one or more of the figures in this paper are available online at http://ieeexplore.ieee.org.

Digital Object Identifier 10.1109/JSTQE.2014.2346613 be generated through the quasi-three-level laser transition process. Low-wavelength lasers can be used for the blue spectrum laser emission combined with the second-harmonic conversion process [7]. For example, the fundamental wavelength of $800 \sim 950 \mathrm{~nm}$ could be transformed into the spectrum of 400 $\sim 445 \mathrm{~nm}$. Compared with semiconductor lasers, it has smaller spectral width and better coherence length, which makes it interest for interferometer experiments. Besides, the quasi-threelevel laser transition around $900 \mathrm{~nm}$ has the potential application as the sources for the differential absorption lidar (DIAL) in water vapor detection, which have wavelengths overlap the absorption peaks of water vapor. [8] As reported in Ref. [4], [9], [10], Nd:CNGG disordered crystal has proved to be an efficient gain medium for the laser oscillation around $930 \mathrm{~nm}$. However, no observation on the laser emissions at $\sim 890 \mathrm{~nm}$ has been reported in Nd:CNGG crystal as of yet.

Waveguide lasers, taking advantages of the compact platform of waveguide structures, are promising integrated light sources [11]-[17]. With appropriate design of dielectric mirrors, lower lasing thresholds and considerable pumping efficiencies can be easily obtained compared with the bulk lasers. The waveguide structure in Nd:CNGG was firstly fabricated by ion implantation, but the propagation loss was high $(3 \mathrm{~dB} / \mathrm{cm})$, which limited the laser emissions from the structure [18], [19].

The femtosecond (fs) laser inscription is a robust, mature technique for waveguide fabrication in a number of gain media, including glasses, ceramics and single crystals [20]-[27]. In crystalline materials, there are three configurations of fs laser inscribed waveguides, i.e., Type I waveguides (with positive refractive index changes in the track), Type II stress-induced waveguides (typically located in the region between two tracks with reduced indices) and Type III depressed cladding waveguides (surrounded by low-index tracks). Type II waveguide was traditionally used for the fabrication of highly efficiency as they provide high index contrast waveguides while creating a minimum lattice distortion at the active volume [28]. In addition, the modal feature of Type II was detected to be a single mode and has smaller size compare with Type III waveguide. Hence, Type II structures are usually superior to those of cladding waveguides. So far, the highest output power of waveguide laser was obtained based on Type II structure [24]. According to our previous work [29], four-level Nd-ion transition from Type III waveguide was observed. But no quasi-three-level laser operation has been observed in the waveguide structure.

In this Letter, we reported on the fabrication of fs laser inscribed Nd:CNGG Type II waveguides. Properties of 


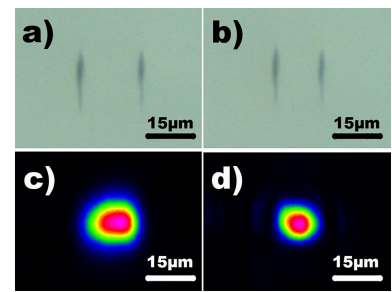

Fig. 1. Cross section of waveguides (W1 and W2) with widths of $20 \mu \mathrm{m}$ (a) and $15 \mu \mathrm{m}$ (b); the measured intensity distribution of the $\mathrm{TM}_{00}$ modes at the wavelength of $\sim 930 \mathrm{~nm}$ (c) and $\sim 890 \mathrm{~nm}$ (d).

waveguide, such as modal profiles and propagation losses, were obtained. Under certain pumping conditions, the stable laser oscillations at low-wavelengths of $\sim 930$ and $\sim 890 \mathrm{~nm}$ were realized inside the waveguides.

\section{EXPERIMENTS OF WAVEGUIDE FABRICATION AND LASER EXCITATION}

The Nd:CNGG crystal used in this paper is the same as the one reported in Ref. [9], which was doped by 2 at.\% $\mathrm{Nd}^{3+}$ ions and cut into dimensions of $8 \times 9.9 \times 1.5(x \times y \times z) \mathrm{mm}^{3}$ with all the facets optically polished. A linearly polarized $120 \mathrm{fs}$ pulsed laser (Spitfire, Spectra Physics, USA) was used for the inscription of damaged tracks, with a central wavelength of $800 \mathrm{~nm}$, a repetition rate of $1 \mathrm{kHz}$ and a maximum pulse energy of $1 \mathrm{~mJ}$. During the inscription process, the energy of the pulses was reduced to $0.84 \mu \mathrm{J}$ and was focused inside the sample with a $40 \times$ microscope objective, scanning the sample at $25 \mu \mathrm{m} / \mathrm{s}$. Two parallel tracks were thus written with different separations. As depicted in Fig. 1(a) and (b), waveguide 1 and waveguide 2 (W1 and W2) were formed between the tracks with width of 20 and $15 \mu \mathrm{m}$, respectively.

Fig. 1(c) and (d) shows the modal profiles of the waveguide at the wavelengths of $\sim 930$ and $\sim 890 \mathrm{~nm}$, respectively, showing single-mode waveguiding features. Adjusting the polarization of the detecting light, we found only TM (parallel to tracks) polarized light could be confined inside the waveguide. To avoid the absorption from the doped rare-earth ions, a laser at wavelength of $632.8 \mathrm{~nm}$ was used to measure the propagation loss by the method described in Ref. [30]. The value of propagation loss was determined to be less than $1 \mathrm{~dB} / \mathrm{cm}$.

We measured the room-temperature luminescence spectra obtained from the W1, W2 and bulk. During the measurement, pumping laser at $810 \mathrm{~nm}$ was coupled into waveguides and the bulk, respectively. Meanwhile, the output light was collected and detected by an optical spectrograph (measurement error less than $2 \mathrm{~nm}$ ). As shown in Fig. 2, shapes of fluorescence spectrum were almost identical. And the value of emission peaks in waveguides was the same as the bulk, which indicates the fs laser inscription process did not affect the fluorescence properties of the Nd:CNGG crystal.

Fig. 3 shows the experimental setup for the laser excitation. Through a convex lens (focal length $20 \mathrm{~mm}$ ), a continuous laser at the wavelength of $810 \mathrm{~nm}$ from a wave tunable Ti:Sapphire laser was coupled into the Nd:CNGG waveguide.

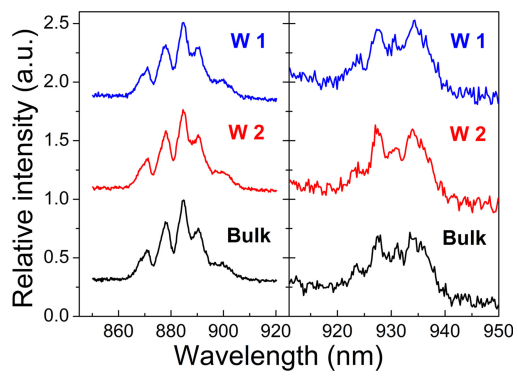

Fig. 2. Luminescence emission spectra of $\mathrm{Nd}^{3+}$ ions at ${ }^{4} \mathrm{~F}_{3 / 2} \rightarrow^{4} \mathrm{I}_{9 / 2}$ transition obtained from W1 (blue solid line), W2 (red dotted line), and the bulk (black dashed line).

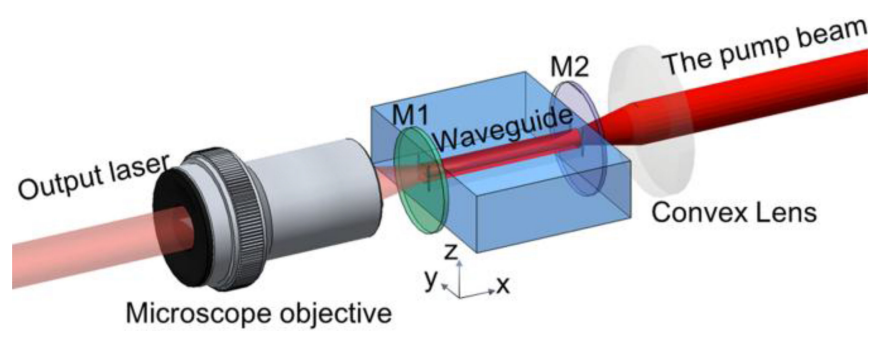

Fig. 3. Experimental setup for the waveguide laser excitation. Mirror 1 (M1) and Mirror 2 (M2) were spectral designed for certain wavelength laser excitation.

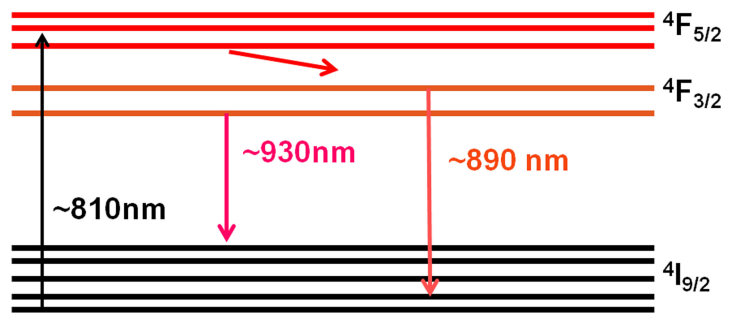

Fig. 4. Schematic description of the energy structure of Nd:CNGG crystal.

To build the resonant optical cavity for the laser oscillation at the low-wavelength, specially designed Mirror 1 and Mirror 2 (M1 and M2) were adhered to the end-facets of the waveguide as the resonator high-reflectivity mirror and out-coupling mirror, respectively.

Fig. 4 depicts the schematic of the pumping together with upper lasing levels of the $\mathrm{Nd}^{3+}$ ions. In the $\mathrm{Nd}$-doped medium, the quasi-three-level transition could induce the lasing at both $\sim 930$ and $\sim 890 \mathrm{~nm}$ corresponding to the emission of ${ }^{4} \mathrm{~F}_{3 / 2} \rightarrow{ }^{4} \mathrm{I}_{9 / 2}$. For CNGG crystal, there are three or four different sites for $\mathrm{Nd}^{3+}$ dopant ions due to the disordered nature of the crystalline host. The coexistence of sites produces broadened fluorescence spectra and distinct fluorescence peaks, which indicates the possibility of multi-wavelength laser generation.

In order to select the laser emission at the certain wavelength, the reflectivity of the mirrors was accurately selected. For example, M1 (M2) has 90\% (>99.98\%) reflectivity at the wavelength of $\sim 930 \mathrm{~nm}$ to realize the laser emission at that wavelength. In addition, a pair of mirrors with $90 \%$ (M1) and $>99.98 \%$ (M2) reflectivity at $\sim 890 \mathrm{~nm}$ was used for the $\sim 890 \mathrm{~nm}$ wavelength laser oscillation. 


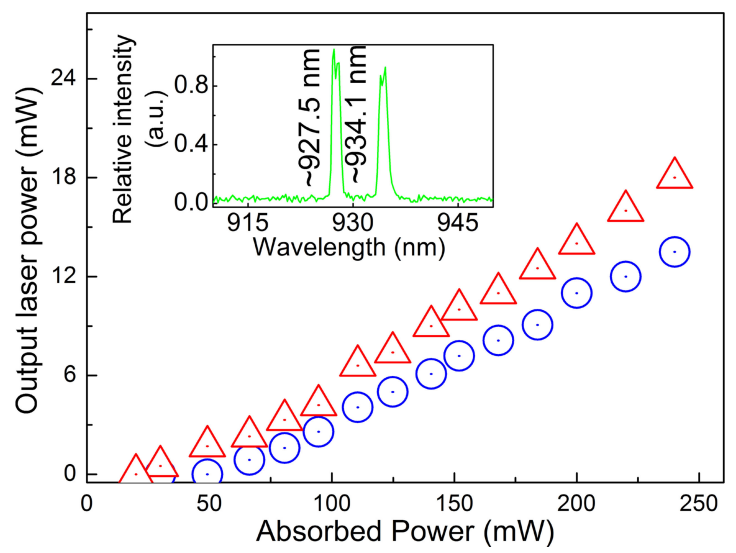

Fig. 5. Power of the total output laser versus the absorbed pumping power from W1 (triangle) and W2 (circle). The inset shows the cw laser oscillation spectra from waveguides after pumping at $810 \mathrm{~nm}$ above the power threshold.

\section{RESULTS AND DISCUSSION}

Using the pair of $\sim 930 \mathrm{~nm}$ mirrors, we obtained the laser oscillation from W1. The inset of Fig. 5 depicts the spectrum of the output laser from $\mathrm{W} 1$ and $\mathrm{W} 2$, when the pumping laser power with the TM polarization was above the laser threshold. As one can see, there is a dual-wavelength laser oscillation at the wavelengths of 927.5 and $934.1 \mathrm{~nm}$. For W1, the laser threshold was around $50 \mathrm{~mW}$ and the maximum output power was around $14 \mathrm{~mW}$ under $\sim 240 \mathrm{~mW}$ absorbed pumping laser. The slope efficiency was around $7.4 \%$. In W2, the laser threshold was decreased to $27 \mathrm{~mW}$ and slope efficiency was $8.5 \%$ indicating the enhanced laser performance induced by smaller volume of the waveguide. Please note the absorbed pump power was evaluated taking into account optics characteristics and the propagation losses, as well as the coupling efficiency of the pump beam into each waveguide ( $\sim 80 \%)$.

Pumping laser with the TE polarization was also used to pumping waveguide laser in this work. However, the slope efficiency of the waveguide laser was only $2 \%$ and the lasing threshold was up to $100 \mathrm{~mW}$. This may because the Type II waveguide is induced by the compression form tracks, which could lead to the birefringence phenomenon in the waveguide region. And light with TM polarization will has higher coupling efficiency compared with TE light. Hence, more pumping light with TM polarization could be coupled into waveguide with the same coupling power.

Compared with the bulk Nd:CNGG laser, an additional wavelength at $\sim 927.5 \mathrm{~nm}$ was found and the threshold was far below the value ( $3 \mathrm{~W}$ ) in the bulk [9]. We believe the enhanced laser operation is induced by the waveguide structure, which has a much smaller volume of the resonant cavity.

The mirrors were then replaced by the pair for $\sim 890 \mathrm{~nm}$. From $\mathrm{W} 2, \sim 890 \mathrm{~nm}$ laser emission was obtained when the power of absorbed laser was more than $70 \mathrm{~mW}$ with TM pumping laser. As depicted in Fig. 6, a dual-wavelength laser oscillation was obtained at the wavelengths of 885 and $891 \mathrm{~nm}$, respectively. The threshold for both wavelengths was similar, and the maximum output power was around $10 \mathrm{~mW}$ when the pumping power

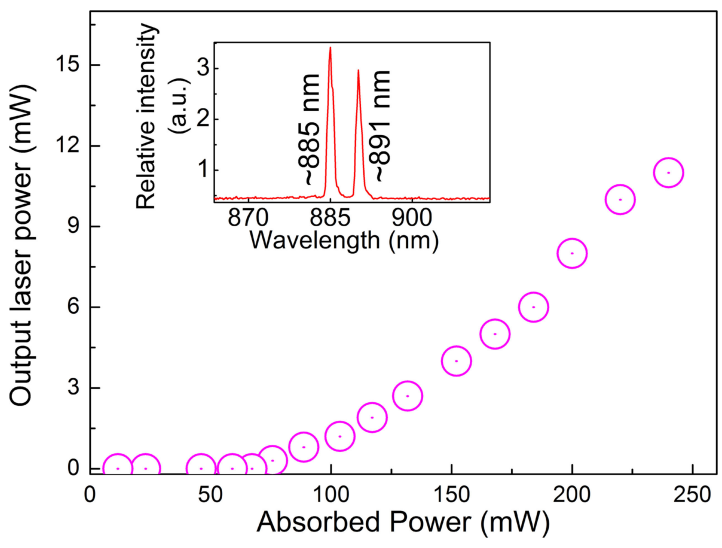

Fig. 6. Output laser power as a function of the absorbed pumping power. The inset is the cw laser oscillation spectra from W2 with pumping at $810 \mathrm{~nm}$ above the pumping power threshold.

was $\sim 240 \mathrm{~mW}$ at the wavelength $810 \mathrm{~nm}$ with TM polarization. This is the first time laser oscillation at $\sim 890 \mathrm{~nm}$ was observed based on Nd:CNGG crystal.

Surprisingly no laser emission at $\sim 890 \mathrm{~nm}$ was found in W1 with the same mirrors and pumping conditions. Similar thing was also observed in Nd:CNGG Type III waveguide [28]. With the same mirrors and pumping conditions, no quasi-three-level laser could be excited. The main difference of these waveguide is the size of volume of the waveguide. It seems that the volume of the waveguide structure directly affects the laser performance for the quasi-three-level wavelength.

To explain this phenomenon, we propose to use the same model as for the three-level laser in Yb-doped materials [31]. In order to achieve laser emission, the gain coefficient should be considered as the main quantity, which is related to the effective cross sections at the laser wavelength $(\lambda)$ including the absorption cross section $\left(\sigma_{\text {abs }}(\lambda)\right)$ and the emission cross section $\left(\sigma_{\mathrm{em}}(\lambda)\right)$. As ground state resonance absorption is present at the laser wavelength for $\mathrm{Nd}$ lasers, the minimum fraction $\left(\beta_{\min , \lambda}\right)$ of excited $\mathrm{Nd}$ ions must balance the ground-state absorption and the gain exactly. For the lowest wavelength, $\beta_{\min , \lambda}$ could be expressed by equation [31]:

$$
\beta_{\mathrm{min}, \lambda}=\frac{\sigma_{\mathrm{abs}}(\lambda)}{\sigma_{\mathrm{abs}}(\lambda)+\sigma_{\mathrm{em}}(\lambda)} .
$$

To achieve transparency at the extraction wavelength, we could calculate the minimum required pump power $\left(P_{\min }\right)$ by the equations

$$
\begin{aligned}
I_{\mathrm{sat}} & =h v /\left(\sigma_{\mathrm{pump}, \mathrm{abs}} \tau_{\mathrm{em}}\right) \\
P_{\mathrm{min}, \lambda} & =\beta_{\mathrm{min}, \lambda} I_{\mathrm{sat}} S
\end{aligned}
$$

where $I_{\text {sat }}$ is the pump saturation intensity parameter; $\sigma_{\text {pump }, \text { abs }}$ and $\tau_{\text {em }}$ are the absorption cross section and the emission lifetime at the pumping wavelength; and $S$ is the waveguide cross section.

For the situation of the quasi-three-level laser transition in Nd:CNGG crystal, $\sigma_{\text {em }}(890)$ and $\sigma_{\text {abs }}(890)$ are $1.8 \times 10^{-20}$ and $0.83 \times 10^{-20} \mathrm{~cm}^{2}$ [5], respectively. According to Eq. (1), 
$\sigma_{\text {abs }}(930)$ is supposed to be less than $0.2 \times 10^{-20} \mathrm{~cm}^{2}$, while $\sigma_{\text {em }}(930)$ is about $0.8 \times 10^{-20} \mathrm{~cm}^{2}$. Hence, $\beta_{\text {min,930 }}$ should be less than 0.18 .

As shown in Eq. (2), the pumping threshold power $\left(P_{\min , \lambda}\right)$ is proportional to $\beta_{\min , \lambda}$ and $S$. Based on the above calculation, the pumping threshold at $\sim 890 \mathrm{~nm}$ should be more than three times larger for laser emission at $930 \mathrm{~nm}$ in the waveguide with the same cross section $S$. Therefore an efficient way to decrease the pumping threshold is to decrease the area of the cross section for the laser emission with higher value of $\beta_{\min , \lambda}$.

The stability of the waveguide laser was discussed. We measured the variation of output laser power by a photodiode detector and an oscilloscope with a sampling interval of $2 \mathrm{~ns}$. The variation ratio of the output light was less than $0.2 \%$ measured over a period of $10 \mathrm{~min}$, which means the $\mathrm{Nd}$ :CNGG waveguide laser is a stable continuous-wave laser source.

In conclusion, low-wavelength laser was generated based on Nd:CNGG waveguides, which was fabricated by the ultrafast laser inscription method. The propagation in the waveguides was single-mode and the measured loss was $1 \mathrm{~dB} / \mathrm{cm}$. The power thresholds of the waveguide lasers was $\sim 50$ and $\sim 70 \mathrm{~mW}$ for the wavelengths of $\sim 930$ and $\sim 890 \mathrm{~nm}$, respectively. The stability of this waveguide laser was discussed and it was proved to be a continuous stable laser. Nevertheless, future effort would be performed on the improvement of the efficiency of the Nd:CNGG waveguide system.

\section{REFERENCES}

[1] Yu. K. Voronko, A. A. Sobol, A. Ya. Karasik, N. A. Eskov, P. A. Rabochkina, and S. N. Ushakov, "Calcium niobium gallium and calcium lithium niobium gallium garnets doped with rare earth ions-effective laser media," Opt. Mater., vol. 20, no. 3, pp. 197-209, 2002.

[2] L. Gheorghe, M. Petrache, and V. Lupei, "Preparation, growth, and characterisation of $\mathrm{Nd}^{3+}$-doped calcium lithium niobium gallium garnet $\left(\mathrm{Nd}^{3+}\right.$ : CLNGG) single crystals," J. Cryst. Growth, vol. 220, no. 1/2, pp. 121-125, 2000.

[3] Yu. K. Voron'ko, N. A. Es'kov, V. V. Osiko, A. A. Sobol', S. A. Sychev, S. N. Ushakov, and L. I. Tsymbal, "Lasing properties of neodymiumdoped calcium-niobium-gallium and calcium-lithium-niobium-gallium garnets at wavelengths of 1.06 and $1.33 \mu \mathrm{m}$," Quantum Electron., vol. 20, no. 6, pp. 494-496, 1993.

[4] Q. N. Li, B. H. Feng, Z. Y. Wei, D. X. Zhang, D. H. Li, Z. G. Zhang, H. J. Zhang, and J. Y. Wang, "Continuous wave $935 \mathrm{~nm} \mathrm{Nd:CNGG} \mathrm{laser} \mathrm{at}$ watt-level power," Opt. Lett., vol. 33, no. 3, pp. 261-263, 2008.

[5] A. Agnesi, S. Dell'Acqua, A. Guandalini, G. Reali, F. Cornacchia, A. Toncelli, M. Toncelli, K. Shimamura, and T. Fukuda, "Optical spectroscopy and diode-pumped laser performance of $\mathrm{Nd}^{3+}$ in the CNGG crystal," IEEE J. Quantum Electron., vol. 37, no. 2, pp. 304-313, Feb. 2001.

[6] Z. B. Shi, X. Fang, H. J. Zhang, Z. P. Wang, J. Y. Wang, H. H. Yu, Y. G. Yu, X. T. Tao, and M. H. Jiang, "Continuous-wave laser operation at $1.33 \mu \mathrm{m}$ of Nd:CNGG and Nd:CLNGG crystals," Laser Phys. Lett., vol. 5, no. 3, pp. 177-180, 2008.

[7] F. Balembois, M. Castaing, E. Herault, and P. Georges, "Low-wavelength emission of Nd-doped lasers," Laser Photon. Rev., vol. 5, no. 5, pp. 659676, 2006.

[8] B. Ileri, C. Czeranowsky, K. Petermann, and G. Huber, "Mixed garnet laser crystals for water vapor detection," in Proc. IEEE Conf. Lasers Electro-Opt. Eur., Europe, 2005, p. 10.

[9] Y. Shi, Q. Li, D. Zhang, B. Feng, Z. Zhang, H. Zhang, and J. Wang, "Comparison of $885 \mathrm{~nm}$ pumping and $808 \mathrm{~nm}$ pumping in Nd:CNGG laser operating at $1061 \mathrm{~nm}$ and $935 \mathrm{~nm}$," Opt. Commun., vol. 283, no. 14, pp. 2888-2891, 2010

[10] K. He, Z. Y. Wei, D. H. Li, Z. G. Zhang, H. J. Zhang, J. Y. Wang, and C. Q. Gao, "Diode-pumped quasi-three-level CW Nd:CLNGG and Nd:CNGG lasers," Opt. Exp., vol. 17, no. 21, pp. 19292-192197, 2009.
[11] C. Grivas, "Optically pumped planar waveguide lasers, Part I: Fundamentals and fabrication techniques," Prog. Quantum Electron., vol. 35, no. 6, pp. 159-239, 2011.

[12] F. Chen and J. R. Vazquez de Aldana, "Optical waveguides in crystalline dielectric materials produced by femtosecond laser micromachining," Laser Photon. Rev., vol. 8, pp. 251-275, 2014.

[13] D. Lancaster, T. Monro, S. Gross, M. Withford, S. Jackson, and H. Ebendorff-Heidepriem, "Efficient $2.9 \mu \mathrm{m}$ fluorozirconate glass waveguide chip laser," Opt. Lett., vol. 38, no. 14, pp. 2588-2591, 2013.

[14] Y. Tan, Y. C. Jia, F. Chen, J. R. V. de Aldana, and D. Jaque, "Simultaneous dual-wavelength lasers at $1064 \mathrm{~nm}$ and $1342 \mathrm{~nm}$ in femtosecond-laserwritten Nd:YVO4 channel waveguides," J. Opt. Soc. Amer. B, vol. 28 , no. 7, pp. 1607-1610, 2011.

[15] R. Mary, G. Brown, S. J. Beecher, F. Torrisi, S. Milana, D. Popa, T. Hasan, Z. P. Sun, E. Lidorikis, S. Ohara, A. C. Ferrari, and A. K. Kar, " $1.5 \mathrm{GHz}$ picosecond pulse generation from a monolithic waveguide laser with a graphene-film saturable output coupler," Opt. Exp., vol. 21, no. 7, pp. 7943-7950, 2013.

[16] M. E. Sánchez-Morales, G. V. Vázquez, E. B. Mejía, H. Márquez, J. Rickards, and R. Trejo-Luna, "Laser emission in Nd:YVO4 channel waveguides at 1064 nm," Appl. Phys. B, vol. 94, no. 2, pp. 215-219, 2009.

[17] C. Zhang, N. Dong, J. Yang, F. Chen, J. R. Vázquez de Aldana, and Q. Lu, "Channel waveguide lasers in Nd:GGG crystals fabricated by femtosecond laser inscription," Opt. Exp., vol. 19, no. 13, pp. 12503-12508, 2011.

[18] L. L. Wang and Y. G. Yu, "Characterization of laser waveguides in Nd:CNGG crystals formed by low fluence carbon ion implantation," Appl. Surf. Sci., vol. 256, no. 8, pp. 2616-2619, 2010.

[19] X. Wang, C. Liu, J. Zhao, and H. Zhang, "Property studies of optical waveguide formed by $\mathrm{keV} \mathrm{He}$-ion implanted into a Nd:CNGG crystal," $J$. Korean Phys. Soc., vol. 55, no. 61, pp. 2638-2641, 2009.

[20] R. Mary, S. J. Beecher, G. Brown, R. R. Thomson, D. Jaque, S. Ohara, and A. K. Kar, "Compact, highly efficient ytterbium doped bismuthate glass waveguide laser," Opt. Lett., vol. 37, no. 10, pp. 1691-1693, 2012.

[21] G. A. Torchia, P. F. Meilán, A. Rodenas, D. Jaque, C. Mendez, and L. Roso, "Femtosecond laser written surface waveguides fabricated in Nd:YAG ceramics," Opt. Exp., vol. 15, no. 20, pp. 13266-13271, 2007.

[22] D. G. Lancaster, S. Gross, A. Fuerbach, H. Ebendorff Heidepriem, T. M. Monro, and M. J. Withford, "Versatile large-mode-area femtosecond laserwritten Tm:ZBLAN glass chip lasers," Opt. Exp., vol. 20, no. 25, pp. 27503-27509, 2012.

[23] Y. Yan, A. Rodenas, F. Chen, R. R. Thomson, A. K. Kar, D. Jaque, and Q. M. Lu, "70\% slope efficiency from an ultrafast laser-written Nd:GdVO4 channel waveguide laser," Opt. Exp., vol. 18, no. 24, pp. 24994-24999, 2010.

[24] T. Calmano, J. Siebenmorgen, O. Hellmig, K. Petermann, and G. Huber, "Nd:YAG waveguide laser with $1.3 \mathrm{~W}$ output power, fabricated by direct femtosecond laser writing," Appl. Phys. B, vol. 100, no. 1, pp. 131-135, 2010.

[25] J. Siebenmorgen, K. Petermann, G. Huber, K. Rademaker, S. Nolte and A. Tünnermann, "Femtosecond laser written stress-induced Nd:Y3A15O12 (Nd:YAG) channel waveguide laser," Appl. Phys. B, vol. 97, no. 2, pp. 251-255, 2009.

[26] T. Calmano, A. Paschke, S. Müller, C. Kränkel, and G. Huber, "Curved Yb:YAG waveguide lasers, fabricated by femtosecond laser inscription," Opt. Exp., vol. 21, pp. 25501-25508, 2013.

[27] F. Reichert, T. Calmano, S. Müller, D.-T. Marzahl, P. W. Metz, and G. Huber, "Efficient visible laser operation of Pr,Mg:SrAl12O19 channel waveguides," Opt. Lett., vol. 38, no. 15, pp. 2698-2701, 2013.

[28] G. A. Torchia1, A. Rodenas, A. Benayas, E. Cantelar, L. Roso, and D. Jaque, "Highly efficient laser action in femtosecond-written Nd:yttrium aluminum garnet ceramic waveguides," Appl. Phys. Lett., vol. 92, no. 11, pp. 111103-1-111103-3, 2008.

[29] Y. Tan, F. Chen, J. R. Vázquez de Aldana, H. Yu, and H. Zhang, "Triwavelength laser generation based on neodymium doped disordered crystal waveguide," Opt. Exp., vol. 21, no. 19, pp. 22263-22268, 2013.

[30] L. Wang, F. Chen, X. Wang, K. Wang, Y. Jiao, L. Wang, X. Li, Q. Lu, $\mathrm{H}$. Ma, and R. Nie, "Low-loss planar and stripe waveguides in $\mathrm{Nd}^{3+}$ doped silicate glass produced by oxygen-ion implantation," J. Appl. Phys., vol. 101, no. 5, pp. 053112-1-053112-4, 2007.

[31] L. D. DeLoach, S. A. Payne, L. L. Chase, L. K. Smith, W. L. Kway, and W. F. Krupke, "Evaluation of absorption and emission properties of $\mathrm{Yb}^{3+}$ doped crystals for laser applications," IEEE J. Quantum Electron., vol. 29 , no. 4, pp. 1179-1191, Apr. 1993. 
Yang Tan received the Ph.D. degree from Shandong University, Shandong, China, in 2011, where he is currently a Lecturer at the School of Physics, Shandong University. His research interests include material modifications by ultrafast lasers and ion beam, nonlinear optics, optical waveguides, etc.

Feng Chen received the Ph.D. degree from Shandong University, Shandong, China, in 2002. He is currently a Professor and the Head of the School of Physics, Shandong University. He was with the Clausthal University of Technology, Germany, from 2003 to 2005, as an Alexander von Humboldt Research Fellow. He became a Professor at Shandong University in 2006. His research interests include material modifications by ultrafast lasers and ion beams, optical waveguides, etc. He is a Fellow of the Institute of Physics, U.K. and a Senior Member of the Optical Society of America and the Chinese Optical Society. He also serves as an Associate Editor of Optical Engineering.

Javier Rodríguez Vàzquez de Aldana received the Bachelor's of Science and $\mathrm{Ph} . \mathrm{D}$. degrees from the University of Salamanca, Spain, in 1997 and 2001, respectively. He is currently an Associate Professor of the Science Faculty, University of Salamanca, Spain. His research activity is focused on the interaction of intense femtosecond pulses with materials and its application to the fabrication of photonic devices. He is a Member of the Laser Microprocessing Research Group, and is also Technical and Scientific Advisor of the Laser Facility at the University of Salamanca.
Haohai Yu was born in Jinan, China, on October 16, 1981. He received the $\mathrm{Ph} . D$. degree from Shandong University, Jinan, China, in 2008. He is currently an Associate Professor at the State Key Laboratory of Crystal Materials and Institute of Crystal Materials, Shandong University. His current research interests include crystal growth, diode-pumped solid-state lasers, and nonlinear optics based on the new crystals.

Huaijin Zhang was born in Shandong, China, in 1965. He received the B.S., M.S., and Ph.D. degrees in physics from Shandong University, Jinan, China, in 1988, 1994, and 2001, respectively. He is currently a Professor of the State Key Laboratory of Crystal Materials, Shandong University. His current research interests include the growth and characterization of different types of crystal materials, including laser, nonlinear, electrooptic, and piezoelectric crystals. 